\title{
Enhancing the Network Lifetime by Deploying Mobile Sink in Wireless Sensor Networks
}

\author{
N. Brindha', S. Vanitha ${ }^{2}$ \\ PG Scholar, Dept of CSE, Dr N.G.P Institute of Technology, Coimbatore, India ${ }^{1}$ \\ Assistant Professor, Dept of CSE, Dr N.G.P Institute of Technology, Coimbatore, India ${ }^{2}$
}

\begin{abstract}
Today Wireless Sensor plays an important role in almost all the field including military surveillance, facility monitoring and environmental monitoring. Wireless Sensor Network (WSN) consists of several nodes which are used to collect and transmit data to the static sink. A static sink acts like an interface between users and the sensor network. The energy utilization of sensor node is essential in the WSN because it manage the lifetime of the sensor network. Data aggregation approach plays a fundamental role in gathering and aggregating data in an energy efficient manner. By tradition tree based approach is more efficient way in transferring the data to the sink. The Mobile sink finds several advantages over static sink. The Proposed work, called Pointed Query Area partition Algorithm with Mobile Sink approach is used. The Mobile sink follows controlled mobility pattern and it can finds the shortest path from its present location for data collection. The proposed algorithms focus on two problems such as lifetime extension and energy consumption. The experimental result of this work is illustrated with below using ns 2 simulation.
\end{abstract}

Keywords: Data aggregation; Mobile sink; Controlled mobility pattern.

\section{INTRODUCTION}

In many fields wireless sensor network plays an important role in capturing and measuring environmental condition such as heat, pressure, humidity and climate from the environmental change. The WSN follows multi hop communication for transmitting data to the sink while using static sink in the network. The sink acts as an interface between the sensor network and user who inject the query into the network. The frequency of data reporting and the number of sensors which report data usually depends on the specific application.The term data aggregation to denote the process of data gathering with aggregation. Data aggregation usually involves the fusion of data from multiple sensors at intermediate nodes and transmission of the aggregated data to the base station.

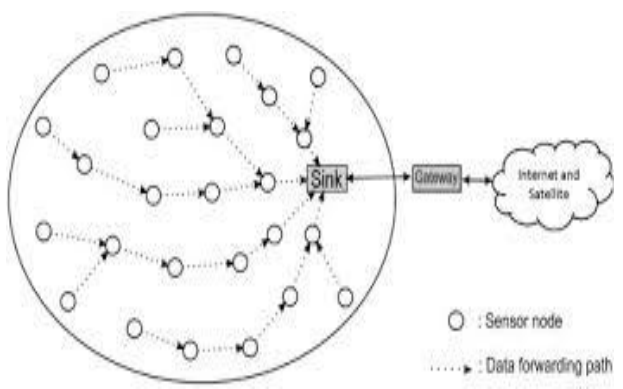

Fig 1.Wireless Sensor Network

Data aggregation attempts to collect the most essential data from the each sensor node and make it available to the sink in an energy efficient manner. In Data aggregation, aggregation function gets performed in each collected data and gives fused information to the sink. The static sink cause high energy utility for transmitting the data hence mobile sink will introduced in order to enhancing the lifetime of the network by reducing the energy utility in data transfer in the sensor network.

\section{LITERATURE SURVEY}

The literature study about the lifetime extension of the sensor network is studied as below.

In the Maximizing Lifetime for the Shortest Path Aggregation Tree in Wireless Sensor Networks paper the authors Luo $\mathrm{D}, \mathrm{Zhu} \mathrm{X}, \mathrm{Wu} \mathrm{X}$, and Chen G study about the problem of finding a shortest path tree with the maximum lifetime when in-network aggregation was used has been studied. The preceding work transformed the problem into the load balancing scheme at each level of the fat tree, proved that the problem was in $\mathrm{P}$, and solved it by min-cost max-flow 
Vol. 6, Issue 6, June 2017

approach in polynomial time. A distributed approach has been proposed for the network [10]. The restriction to shortest path tree comes from the requirement of delay. The results show that their approach greatly improves the lifetime of the network.

In the paper Supporting Aggregate Queries over Ad-Hoc Wireless Sensor Networks the author Madden S, Szewczyk R, Franklin M J, and Culler D deals the different techniques for applying database style aggregates with groups to sensor readings flowing through ad-hoc sensor networks. By applying generic aggregation operations in the tradition of database systems, this approach offers the ability to query arbitrary data in a sensor network without custom-building applications.. Finally, by snooping on messages in the shared channel and applying techniques for hypothesis testing, the model was able to substantially improve the performance of their basic approach [15].

In the paper Aggregation Tree Construction in Sensor Networks the author Ding M,Cheng X, and Xue G says the heuristics to construct and maintain an energy-aware aggregation tree in sensor networks. Applying this tree structure, network lifetime can be extended by turning off the radios of all leaf nodes, and by restricting the post of query and the dissemination of data along this reversed broadcast tree. This model also simulated and analysed the performance of EADAT algorithm in terms of network lifetime, energy saving, data delivery ratio and the protocol overhead. Simulation results show that EADAT performs very well [4].

The paper Building Maximum Lifetime Shortest Path Data Aggregation Trees in Wireless Sensor Networks authors Shan Mengfan, Chen Guihai, Luo Dijun, et al says that the model of the problem of finding a shortest path tree with the maximum lifetime when in-network aggregation was used. The model have to transformed the problem into the load balancing scheme at each level of the fat tree, proved that the problem was in $\mathrm{P}$, and solved it by a centralized approach in polynomial time. The work extended their discussion to WSNs without aggregation and present interesting theoretical results [20].

The Paper Maximizing Lifetime for Data Aggregation in Wireless Sensor Networks author Yuan Xue, Yi Cui, and Klara Nahrstedt tells about the Wireless sensor network deals with the problem of maximizing lifetime routing for data aggregation in sensor networks. Inspired by Garg-Konemann algorithm for multi-commodity flow, this paper presents a novel tree-based approximation algorithm, which addresses the unique traffic characteristic of data aggregation and achieves faster running time.[29].The main contributions of this paper were as follows. First, it extends the framework of multi commodity flow problem to address the unique characteristic of data aggregation in network lifetime maximization. The proposed tree-based algorithm was shown to greatly reduce the running time, compared to existing algorithms, and achieve better scalability in terms of network size than existing approach. Second, based on this improved algorithm, this paper studied the network lifetime maximization problem when multiple data sinks exist in data aggregation and evaluate the impact of number of sinks on the network lifetime.

\section{STATIC VS MOBILE SINK IN WSN}

\section{A. Static Sink}

In the early days, a typical WSN was composed of static sensor nodes and a static sink placed inside the observed region. In such a setup, the major energy consumer is the communication module of each node. In practice, multi-hop communication is required for sending data from sources to sink nodes. Consequently, the energy consumption depends on the communication distance between the two sensor nodes. Adding some mobile sinks to a set of static sinks has been shown to improve the data delivery rate and to reduce energy dissipation of the sensor nodes.

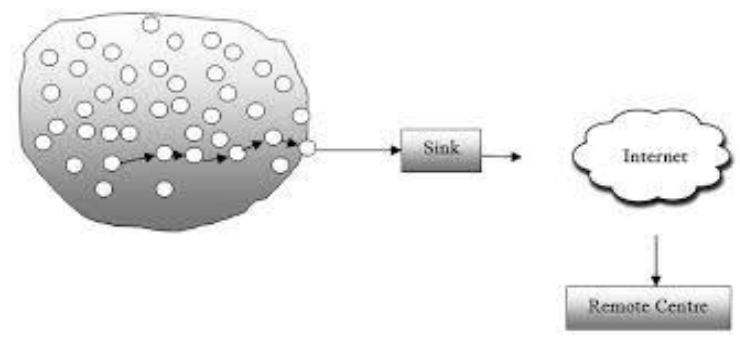

Fig 2. Static sink in WSN

\section{B. Mobile Sink}

Another approach for extending the lifetime of the network is the utilization of a mobile sink. The Mobile sink is to save the sensor energy for multi-hop communication in transferring data in WSN. A mobile sink can follow different types of mobility patterns in the sensor field, such as random mobility, predictable/fixed path mobility, or controlled 
mobility, which has consequences with respect to energy efficiency and data collection strategies. Here the mobile sink follows the controlled mobility pattern in which sink can be controlled or guided by certain query.

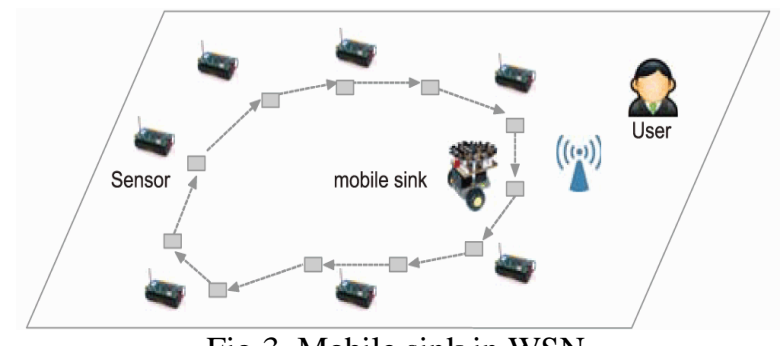

Fig 3. Mobile sink in WSN

C. Comparison Between Static And Mobile Sink

The following table represents the overall comparison between the static and mobile sink.

TABLE I COMPARISON TABLE

\begin{tabular}{|l|l|l|}
\hline Parameter & Static sink & Mobile sink \\
\hline Reliability & Medium & High \\
\hline Energy Expenditure & Medium & High \\
\hline Latency & Low & Medium \\
\hline Throughput & Medium & High \\
\hline Network Lifetime & Medium & High \\
\hline
\end{tabular}

\section{OPTIMAL QUERY AREA PARTITION ALGORITHM WITH MOBILE SINK}

The main challenges in wireless sensor network are energy consumption, network lifetime and security. Though higher utilization of energy in sensor node cause the minimized network lifetime.

In the Optimal Query Area Partition Algorithm with Mobile Sink approach is mainly involved in reducing the energy utility for data transmission. In this algorithm, the network area is partition into sub region. In each region one node is selected as an aggregator node. Based on the user's query the aggregator node will collect the data from all remaining node in the network. The aggregator node performs the aggregation function to reduce the number of transmission.

The Mobile sink is used to save sensor energy for multihop communication in transferring data to the sink. It follows the controlled mobility pattern. Here the sink can be controlled or guided by certain parameters or query. By following the user's query the mobile sink will finds the shortest path from its present location using the Efficient Path Selection algorithm. The mobile sink will reduce the communication cost and energy utilization for transmitting the data to the sink. Hence lifetime of the sensor network can get maximized.

Using Mobile sink in wireless sensor network provides security and it also prevent from information hacker.

\section{A. STEPS}

In this algorithm, four steps can take place: (a) Query formation (b) Network Region Division (c) Selection of aggregation nodes (d) Data aggregation (e) Mobile sink and Data collection process.

\section{a. Query Formation}

The user will allows to posing declarative queries $Q$ over sensor networks which is specified as a sextuple. It adheres to the Tiny-SQL query language. Tiny-SQL query language is a query processing system for extracting information from a network.

For example, Select nodeid, temperature from sensors where temperature $>35$ sample period $4 \mathrm{~s}$.

b. Network Region Division

After Submitting the query in the sensor network, the network area is divided into sub region using Geographical routing protocol as shown in Fig 5. This protocol formulates an efficient route search towards the destination.

c. Selection of aggregation node and tree construction

After dividing the network region, the aggregation node selection will be performed. The sink node sends query message to the close neighbourhood nodes of each query sub region are called root nodes. A root node sends a message to entire queried region as in Fig 6. From that message the aggregation root's level is assigned which depends on how 
many hops away from the root. Initially the aggregation root's level is zero. A node without this level can receives this message then its own level to be plus one with their respective message level.

\section{d. Data aggregation}

The node which is closest to the aggregation root is chosen as parent node. Based on the query message, a number of routing tree has been constructed with the aggregation root node. The aggregation node will perform aggregation function of each collected data from the entire aggregator node.

e. Mobile Sink and Data Collection Process

The Mobile sink follows controlled mobility pattern in which it finds the shortest path from its present location to collect the fused data from every individual aggregator node in Fig 9.

\section{EXPERIMENTAL ANALYSIS}

The result runs in NS2 stimulation framework using Fedora environment. Network Simulator (NS) is a simulation tool targeted at both wired and wireless (local and satellite) networking research. NS is a very promising tool and is being used by universities and researchers The experimental analysis was illustrated as below

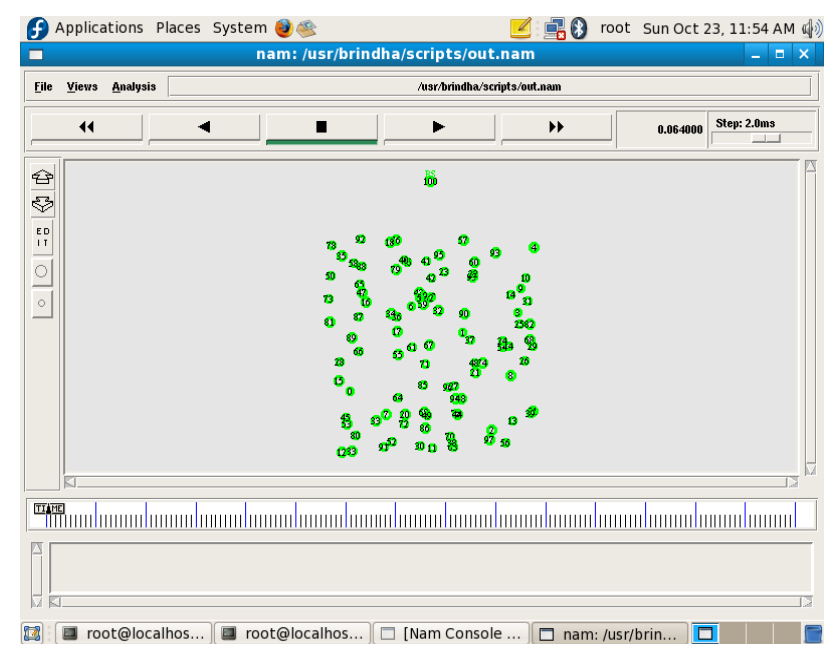

Fig 4. Deployment of sensor nodes

By using Random Way Point (RWP) model the nodes are deployed in the network as in Fig 4.In this model the mobile nodes moves randomly and freely without restriction.

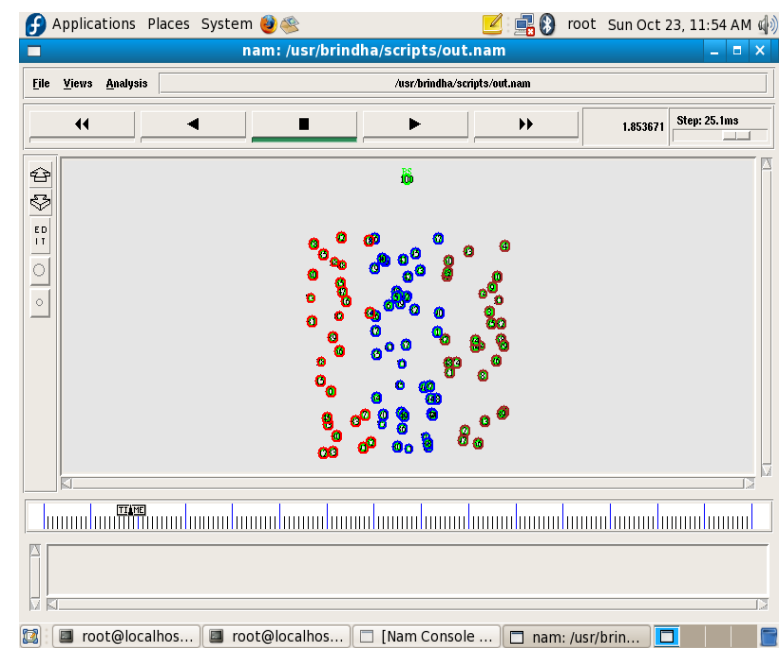

Fig 5. Dividing the region into sub region

In the Fig 5 the sensor area is divided into sub region by using geographical routing protocol. The Geographical routing protocol uses location information to formulate an efficient route search towards the destination. 


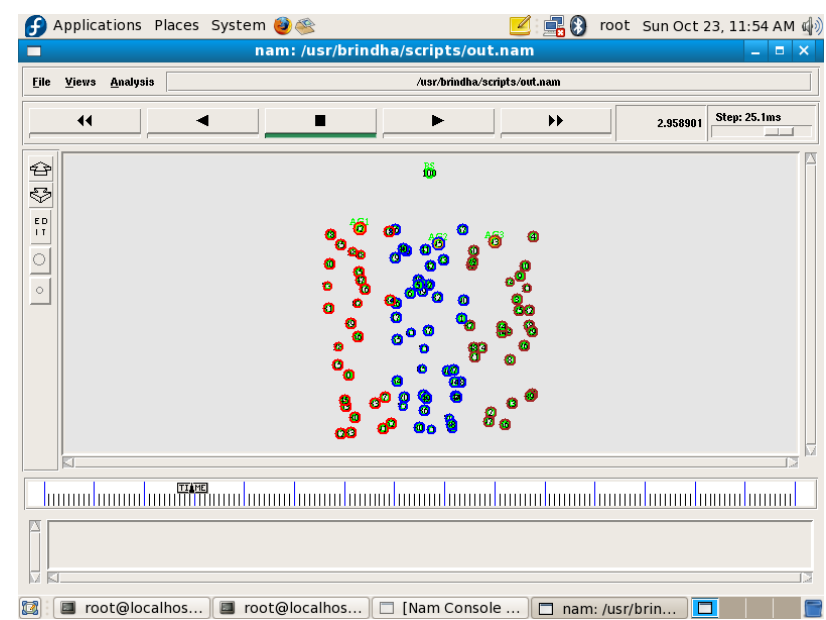

Fig 6. Selection of aggregation node

The Fig 6 indicates the selection of aggregation node which performs aggregation function in each sub region of the sensor network.

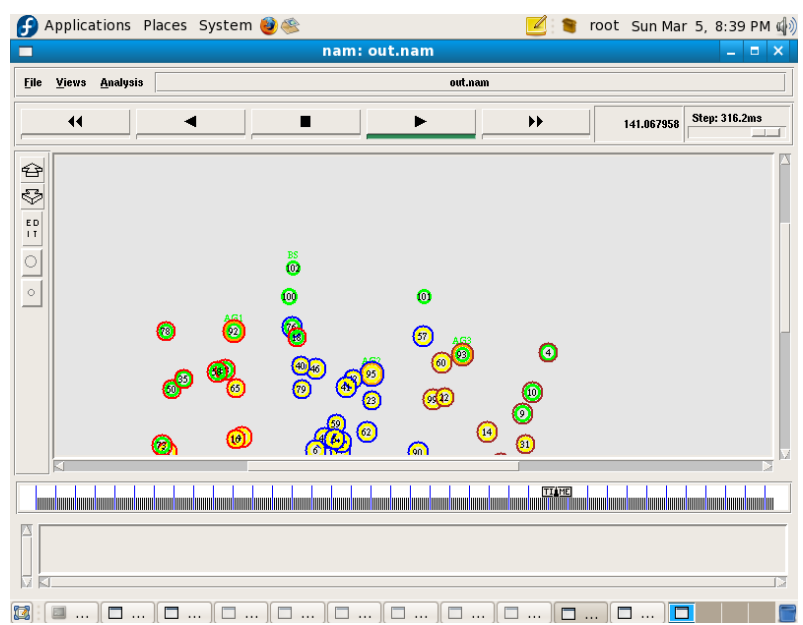

Fig 7. Mobile Sink

In the Fig 7 Mobile sink follows the controlled mobility pattern in which it finds the shortest path from its present location to collect the fused data from every individual aggregator node.

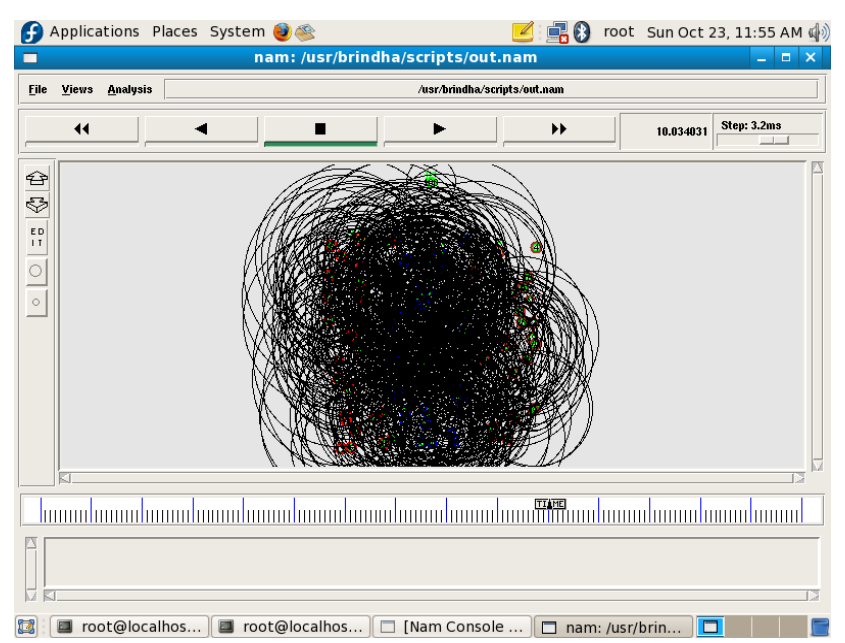

Fig 8. Communication between nodes

In the Fig 8 indicates the communication between the aggregation node and normal nodes in the network. 
Vol. 6, Issue 6, June 2017

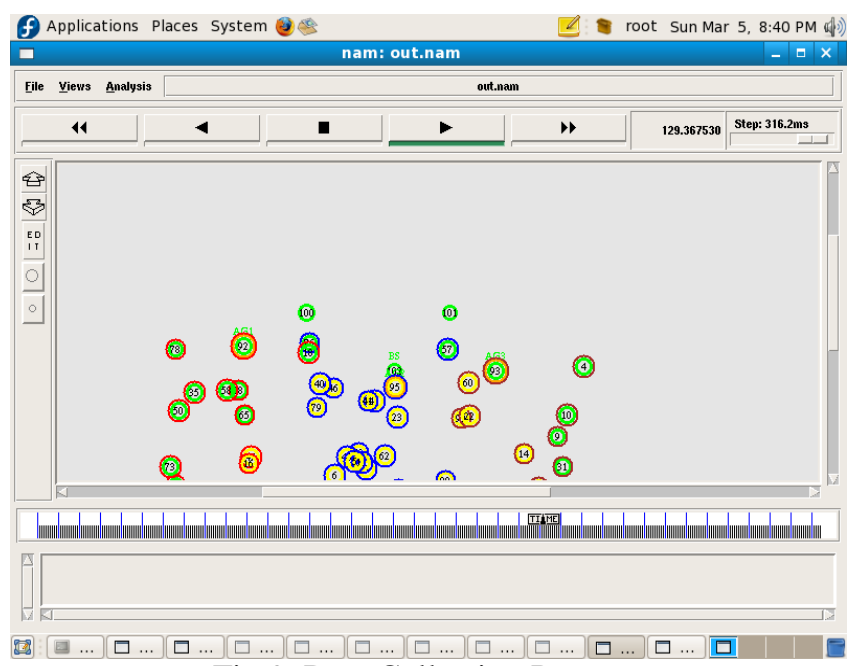

Fig 9. Data Collection Process

The above Fig 9 indicates that the mobile sink collect the fused data from every individual aggregator node. The data collection process used to transmit data, receive data, and listen for the energy minimization, and energy consumption of each node to reach consensus, thus extending the system life cycle.

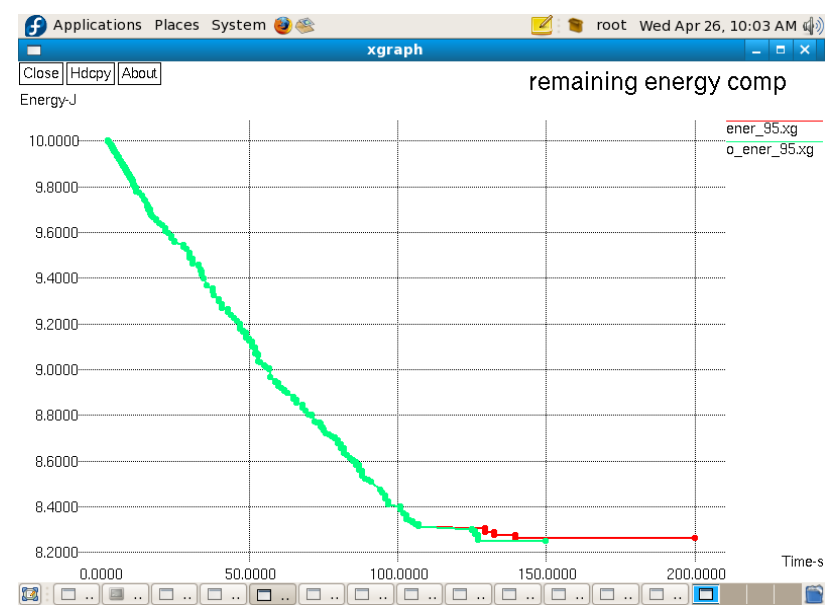

Fig 10 Energy Consumption graph

The Fig 10 indicates the comparison graph between the static and mobile sink. Here Green colour indicates the static sink and the Red colour indicates the mobile sink. The mobile sink use minimum energy utility of data transfer.

\section{CONCLUSION}

The Optimal Query Area Partition algorithm with Mobile sink approach has been used. This algorithm involved in partition of query area, aggregation node selection and energy efficient aggregation tree construction is carried over. The Mobile sink uses the efficient path selection algorithm for finding the shortest path from its present location.

Though these approaches are well energy constrained in collecting the data from the aggregator node and hence the network lifetime gets maximized. The security can also provide for the local communication between the aggregation nodes and mobile sink. Thus the data can get prevented from information hackers.

\section{REFERENCES}

[1] Chen Jia, Shaohua Wan and Yudong Zhang(2016), 'On the Construction of Data Aggregation Tree with Maximizing Lifetime in Large Scale Wireless Sensor Networks', IEEE Sensors Journal,pp.1-7.

[2] Bahram Alinia, Mohammad Hassan Hajiesmaili, Ahmad Khonsari (2015), 'On the construction of maximum-quality aggregation trees in deadline-constrained WSNs'. INFOCOM 2015,pp. 226-234.

[3] Cristescu R,Beferull-Lozano B, and Vetterli M (2004), 'On network correlated data gathering', in IEEE INFOCOM.

[4] Ding M,Cheng X, and Xue G(2003), 'Aggregation Tree Construction in Sensor Networks', in Proceedings of the 58th IEEE Vehicular Technology Conference, vol. 4, pp. 2168-2172. 


\section{IJARCCE}

\section{International Journal of Advanced Research in Computer and Communication Engineering}

ISO 3297:2007 Certified

Vol. 6, Issue 6, June 2017

[5] Giridhar and Kumar P R(2005), 'Computing and communicating functions over sensor networks', IEEE Journal on Selected Areas in Communications, vol. 23, pp. 755-764.

[6] Heinzelman W, Chandrakasan A, Balakrishnan H(2002), 'An application-specific protocol architecture for wireless microsensor networks'. IEEE Trans on Wireless Communications,pp. 660-670.

[7] He Jing, Ji Shouling, Pan Yi, et al.(2014), 'Constructing load-balanced data aggregation trees in probabilistic wireless sensor networks'. IEEE Trans on Parallel and Distributed Systems, pp. 1681-1690.

[8] Intanagonwiwat C, Estrin D, Govindan R, and Heidemann J(2002), 'Impact of Network Density on Data Aggregation in Wireless Sensor Networks', in Proceedings of 22nd International Conference on Distributed Computing Systems, pp. 457-458.

[9] Johannes Niedermayer, Mario A. Nascimento, Matthias Renz, et al.(2014), 'Continuous Quantile Query Processing in Wireless Sensor Networks'. in Proc. 17th International Conference on Extending Database Technology (EDBT), pp. 247-258, Athens, Greece.

[10] Luo D,Zhu X,Wu X, and Chen G(2011), 'Maximizing lifetime for the shortest path aggregation tree in wireless sensor networks', in IEEE INFOCOM

[11]Liu C and Cao G (2010), 'Distributed monitoring and aggregation in wireless sensor networks', in IEEE INFOCOM.

[12] Li J,Deshpande A, and Khuller S(2010), 'On computing compression trees for data collection in wireless sensor networks', in IEEE INFOCOM.

[13] Lu Wang, Robert Christensen, Feifei Li, Ke Yi(2016), 'Spatial Online Sampling and Aggregation', In Proceedings of 42nd International Conference on Very Large Data Bases (VLDB 2016), pp. 1-12, New Delhi, India.

[14] Mainwaring A,Culler D,Polastre J,Szewczyk R, and Anderson J(2002), 'Wireless sensor networks for habitat monitoring', in ACM WSNA.

[15] Madden S,Szewczyk R,Franklin M J,and Culler D(2004), 'Supporting Aggregate Queries Over Ad-Hoc Wireless Sensor Networks', in Proceedings of the 4th IEEE Workshop on Mobile Computing Systems and Applications, pp. 49-58.

[16] Manjhi A, Nath S, Gibbons P B.(2005), 'Tributaries and deltas: Efficient and robust aggregation in sensor network streams', in Proceedings of SIGMOD 2005. New York: ACM,pp. 287-298.

[17] Mingwang Tang, Feifei Li(2014), 'Scalable Histograms on Large Probabilistic Data', In Proceedings of 20th ACM SIGKDD International Conference on Knowledge Discovery and Data Mining (SIGKDD 2014), pp. 631-640, NYC, NY.

[18] Qin Zhang,Li F, Ke Yi(2008), 'Finding Frequent Items In Probabilistic Data', In Proceedings of 27th ACM SIGMOD International Conference on Management of Data (SIGMOD 2008), pp. 819-832, Vancouver, Canada.

[19] Robert Christensen, Lu Wang, Feifei Li, et al(2005), 'STORM: Spatio-Temporal Online Reasoning and Management of Large Spatio-Temporal Data', In Proceedings of 34th ACM SIGMOD International Conference on Management of Data (SIGMOD 2015), pp. 1111-1116, Melbourne, Australia.

[20]Shan Mengfan, Chen Guihai, Luo Dijun, et al.(2014), 'Building maximum lifetime shortest path data aggregation trees in wireless sensor networks', ACM Trans on Sensor Networks,.pp. 1-24.

[21] Shrivastava N,Buragohain C,Agrawal D, and Suri S(2004), 'Medians and beyond: new aggregation techniques for sensor networks', in Proceedings of the 2nd International Conference on Embedded Networked Sensor Systems (SenSys '04), pp. 239-249, ACM, New York, NY, USA.

[22]Tung-Wei Kuo, Kate Ching-Ju Lin, Ming-Jer Tsai(2014), 'On the Construction of Data Aggregation Tree with Minimum Energy Cost in Wireless Sensor Networks: NP-Completeness and Approximation Algorithms'. CoRR abs/1402.6457.

[23]Wu Y,Fahmy S, and Shroff N B(2008), 'On the construction of a maximum-lifetime data gathering tree in sensor networks: NP-Completeness and approximation algorithm', in IEEE INFOCOM.

[24]Wan Shaohua(2014), 'Energy-efficient adaptive routing and context-aware lifetime maximization in wireless sensor networks'. International Journal of Distributed Sensor Networks.

[25]Wei Huang, Lixin Ding(2012), 'The Shortest Path Problem on a Fuzzy Time-Dependent Network'; IEEE Transactions on Communications, Volume 60, pp. $3376-3385$.

[26]Wei Huang, Jinsong Wang(2016), 'The shortest path problem on a time-dependent network with mixed uncertainty of randomness and fuzziness', IEEE Transactions on Intelligent Transportation Systems, Volume PP, pp. 1-11.

[27]Wei Huang, Sung-Kwun Oh, Zhaolu Guo, Witold Pedrycz(2013), 'A space search optimization algorithm with accelerated convergence strategies', Applied Soft Computing, Volume 13,pp. 4659-4675.

[28]Xu N,Rangwala S,Chintalapudi K K,Ganesan D, Broad A,Govindan R, and Estrin D(2004), 'A wireless sensor network for structural monitoring', in ACM SenSys.

[29]Yuan Xue, Yi Cui, and Klara Nahrstedt(2005), 'Maximizing lifetime for data aggregation in wireless sensor networks'. Mobile Networks and Applications, 10(6), pp.853-864.

[30]Yu B,Li J, and Li Y(2009), 'Distributed data aggregation scheduling in wireless sensor networks', in IEEE INFOCOM. 